\title{
Pull vs. wet: rendimiento diagnóstico y calidad de las muestras de las PAAF guiadas por USE en las masas sólidas del páncreas
}

\author{
Cecilia Curvale, ${ }^{1}$ Ignacio Málaga, ${ }^{1}$ Paloma Rojas Saunero, ${ }^{2}$ Viviana Tassi, ${ }^{3}$ Enrique Martins, ${ }^{4}$ \\ Hui Jer Hwang ${ }^{1}$ \\ ${ }^{1}$ Servicio de Gastroenterología y Endoscopia, Hospital El Cruce, Florencio Varela, Argentina. \\ ${ }^{2}$ Erasmus Medical Center, Róterdam, Paises Bajos. \\ ${ }^{3}$ Servicio de Anatomía Patológica, Hospital El Cruce, Florencio Varela, Argentina. \\ ${ }^{4}$ Servicio de Laboratorio, Hospital El Cruce, Florencio Varela, Argentina.
}

Acta Gastroenterol Latinoam 2021;51(1):42-51

Recibido: 01/02/2021 / Aceptado: 16/02/2021 / Publicado online: 22/03/2021 / https://doi.org/10.52787/knpg3537

\section{Resumen}

El diagnóstico diferencial de las masas pancreáticas es un desafio. El método de punción aspirativa con aguja fina guiado por ultrasonografía endoscópica, de mayor rendimiento diagnóstico, no se ha establecido. Se realizó un estudio prospectivo, aleatorizado, de doble ciego de punción aspirativa con aguja fina, guiada por ultrasonografía endoscópica, en las lesiones sólidas del páncreas para comparar y evaluar el rendimiento diagnóstico y la calidad del aspirado entre la técnica wet y pull. Se enrolaron 41 pacientes. La técnica wet presentó una sensibilidad, una especificidad, un valor predictivo positivo y negativo y una precisión diagnóstica de: 58,3\%; 100\%; 100\%; 25\% y 63,4\%, respectivamente. Por su parte, en la técnica pull fueron: 75\%; 100\%; 100\%; $35,7 \%$ y 78,1\%. Comparando el rendimiento diagnóstico entre ambas técnicas, no hubo diferencia estadisticamente

Correspondencia: Cecilia Curvale

Correoelectrónico: cecicurvale@hotmail.com significativa (test de McNemar, $p=0,388$ ). Respecto a la celularidad del espécimen, tanto en muestras de citología como en el bloque celular, no se observaron diferencias relevantes entre las técnicas ( $p=0,84$ y 0,61, respectivamente). Sobre la presencia de sangre contaminante, no hubo diferencias en las muestras de citología $(p=0,89)$ y tampoco en las de bloque celular $(p=0,08)$. La adecuación de las muestras de citología para el diagnóstico fue similar en ambas técnicas (wet $=57,5 \%$ y pull $=56,7 \%, p=0,94)$ y tampoco hubo diferencias en el bloque celular (wet $=75 \%$ y pull $=66,1 \%$, $p=0,38)$. En este estudio no observamos diferencias en el rendimiento diagnóstico ni en la calidad de la muestra entre las técnicas comparadas. Dado que ambos procedimientos son efectivos, se sugiere la utilización simultánea y alternada de ambos métodos.

Palabras claves. Cáncer de páncreas, punción aspirativa con aguja fina, ecoendoscopía.

\section{Pull vs. Wet: Diagnostic Performance and Sample Quality of EUS-Guided FNA in Solid Lesions of Pancreas}

\section{Summary}

Differential diagnosis of pancreatic masses is challenging. The endoscopic ultrasound-guided fine-needle aspiration method with the highest diagnostic yield has not been esta- 
blished. It was realized a prospective, randomized, doubleblind study of the endoscopic ultrasound-guided fine-needle aspiration in solid lesions of the pancreas to compare and evaluate diagnostic yield and aspirate quality between wet and pull technique. Forty-one patients were enrolled. The wet technique presented a sensitivity, a specificity, a positive and negative predictive value, and a diagnostic accuracy of $58.3 \%, 100 \%, 100 \%, 25 \%$ and $63.4 \%$, respectively. In the capillary technique they were: 75\%, 100\%, 100\%, $35.7 \%$ and $78.1 \%$, respectively. Comparing the diagnostic yield between both techniques, there was no statistically significant difference (McNemar's test $p=0.388$ ). Regarding the cellularity of the specimen, both in cytology and the cell block samples, no significant difference was observed between the techniques ( $p=0.84$ and 0.61 , respectively). With respect to contaminating blood in the specimen, there was no difference in cytology samples $(p=0.89)$ and no difference in cell block samples $(p=0.08)$. The suitability of cytology samples for diagnosis was similar in both techniques (wet = $57.5 \%$ and capillary $=56.7 \%, p=0.94)$ and there was no difference in cell block samples (wet $=75 \%$ and capillary $=$ $66.1 \%, p=0.38$ ). In this study we did not observe differences in diagnostic yield or sample quality. Since both techniques are effective, we suggest the simultaneous and alternate use of both methods.

Keywords. Pancreatic cancer, fine needle aspiration, echo endoscopy.

\section{Abreviaturas}

PAAF: Punción aspirativa con aguja fina.

USE: Ultrasonografía endoscópica.

RIN: Razón internacional normatizada.

HJH: Hui Jer Hwang.

DS: Desvio estándar.

RMN: Resonancia magnética nuclear.

IgG: Inmunoglobulina $G$.

LR+: Likelihood ratio positivo.

H\&E: Hematoxilina y eosina.

TC: Tomografía computada.

\section{Puntos clave}

- La USE se ha posicionado como una herramienta imprescindible en el proceso de diagnóstico, en la estadificación y en la punción de las lesiones sólidas de páncreas.

- En este estudio no observamos diferencias significativas en el rendimiento diagnóstico ni en la calidad de las muestras entre las técnicas de punción wet y pull.
- Dado que ambos procedimientos son efectivos, se sugiere la utilización simultánea y alternada de estos en las punciones de lesiones sólidas de páncreas guiadas por la USE.

\section{Introducción}

El diagnóstico diferencial de las masas pancreáticas es un desafío clínico. Las decisiones terapéuticas respecto a estos pacientes se apoyan, principalmente, en lograr diagnosticar o excluir la malignidad. ${ }^{1}$ Por lo tanto, el diagnóstico anatomopatológico adquiere relevancia para optimizar el manejo de estos pacientes. ${ }^{2}$ Un diagnóstico exacto evitaría procedimientos innecesarios en los individuos con patologías benignas y ayudaría a identificar de manera más precisa a quienes se podrían beneficiar con una intervención quirúrgica o un tratamiento oncológico. ${ }^{3}$

La punción aspirativa con aguja fina (PAAF), guiada por la ultrasonografía endoscópica (USE), se ha transformado en una herramienta indispensable para la adquisición de material citológico e histológico en el diagnóstico de estas lesiones. Los estudios de revisión sistemática y metaanálisis han demostrado que la USEPAAF tiene una sensibilidad del $85-91 \%$ y una especificidad del 94-98\%.3., ${ }^{3}$ Por su seguridad, rendimiento y precisión diagnóstica, la USE-PAAF ha reemplazado progresivamente a la PAAF percutánea en los centros que disponen de ella. ${ }^{5}$

Existen diferentes técnicas de punción, pero el método de mayor rendimiento diagnóstico aún no ha sido establecido. La variante considerada como estándar consiste en la punción de la lesión sólida del páncreas con una aguja biselada con estilete y la aplicación de succión al vacío con una jeringa. ${ }^{6}$

En el último tiempo, se han desarrollado nuevas técnicas con el objetivo de incrementar la precisión y el rendimiento diagnóstico. Una de ellas consiste en retirar de forma continua y lenta el estilete de la aguja mientras se están realizando los movimientos de la punción, sin usar una jeringa de succión para crear una mínima presión negativa (a menor presión negativa, menor contaminación con sangre). Esta técnica se denomina: "aspiración por capilaridad" o pull. ${ }^{7}$

Otro método novedoso es el denominado "técnica de succión húmeda" o wet: consiste en utilizar la aguja de punción sin estilete e inyectar, dentro del canal de la aguja, $5 \mathrm{ml}$ de solución fisiológica para reemplazar la columna de aire con agua. Durante la punción, se coloca una jeringa con presión negativa de $10 \mathrm{ml}$ para generar la succión de la lesión. Se cree que al reemplazar la columna de aire por la de agua, esta transmitiría mejor la presión 
negativa ya que, basado en los principios de la dinámica de los fluidos, el agua es menos compresible que el aire. Además, el agua "lubricaría" la superficie interna de la aguja y podría disminuir la fricción entre el tejido aspirado y la pared por tener un efecto de capilaridad. ${ }^{6,8}$

Los estudios que describieron estas nuevas técnicas siempre tomaron como referencia el método estándar y obtuvieron mejores porcentajes de sensibilidad/especificidad y muestras de mayor calidad. ${ }^{7} 8$ Sin embargo, no se han comparado entre sí. Los objetivos de este estudio fueron evaluar y comparar el rendimiento diagnóstico y la calidad del aspirado entre ambos métodos de punción.

\section{Materiales y métodos}

Diseño. Se realizó un estudio prospectivo, aleatorizado, simple ciego para evaluar y comparar el rendimiento diagnóstico y la calidad de las muestras entre dos técnicas de PAAF guiadas por la USE en lesiones sólidas de páncreas: 1) método húmedo o wet, 2) método de capilaridad o pull (Figura 1).

Dado que en cada uno de los pacientes se aplicaron ambos métodos de punción de manera alternada, se realizó la aleatorización de la técnica en el inicio, a fin de evitar diferencias de calidad generadas por el orden de punción. La aleatorización fue simple, mediante una tabla electrónica en una planilla de cálculo Excel@.

Durante todo el protocolo, el método wet se conoció como la técnica nro. 1 y el método pull como la técnica nro. 2.

La anatomopatóloga desconocía la técnica de punción efectuada con la que se había obtenido cada una de las muestras (simple ciego). Se ubicaba en la sala contigua al quirófano. La asistente acercaba de manera sucesiva las mismas para su evaluación in situ y comunicaba los resultados de la revisión (respecto a tener o no material suficiente) al endoscopista. Los informes finales de la anatomopatóloga (que se completaban en el servicio de Anatomía Patológica) eran descripciones sobre la calidad de la muestra y el diagnóstico para cada uno de los frascos (en el orden en los que habían sido obtenidos).

Ámbito y población. Se enrolaron en forma consecutiva, aleatoria y con periodicidad igual a 1 , a todos los pacientes derivados al servicio de Gastroenterología del Hospital El Cruce para la evaluación y la PAAF guiada por la USE de las lesiones sólidas del páncreas diagnosticadas por una tomografía computada, resonancia magnética o las USE previas. Se incluyeron a los pacientes desde enero a diciembre de 2018.

Figura 1. Diagrama de flujo del diseño de este estudio

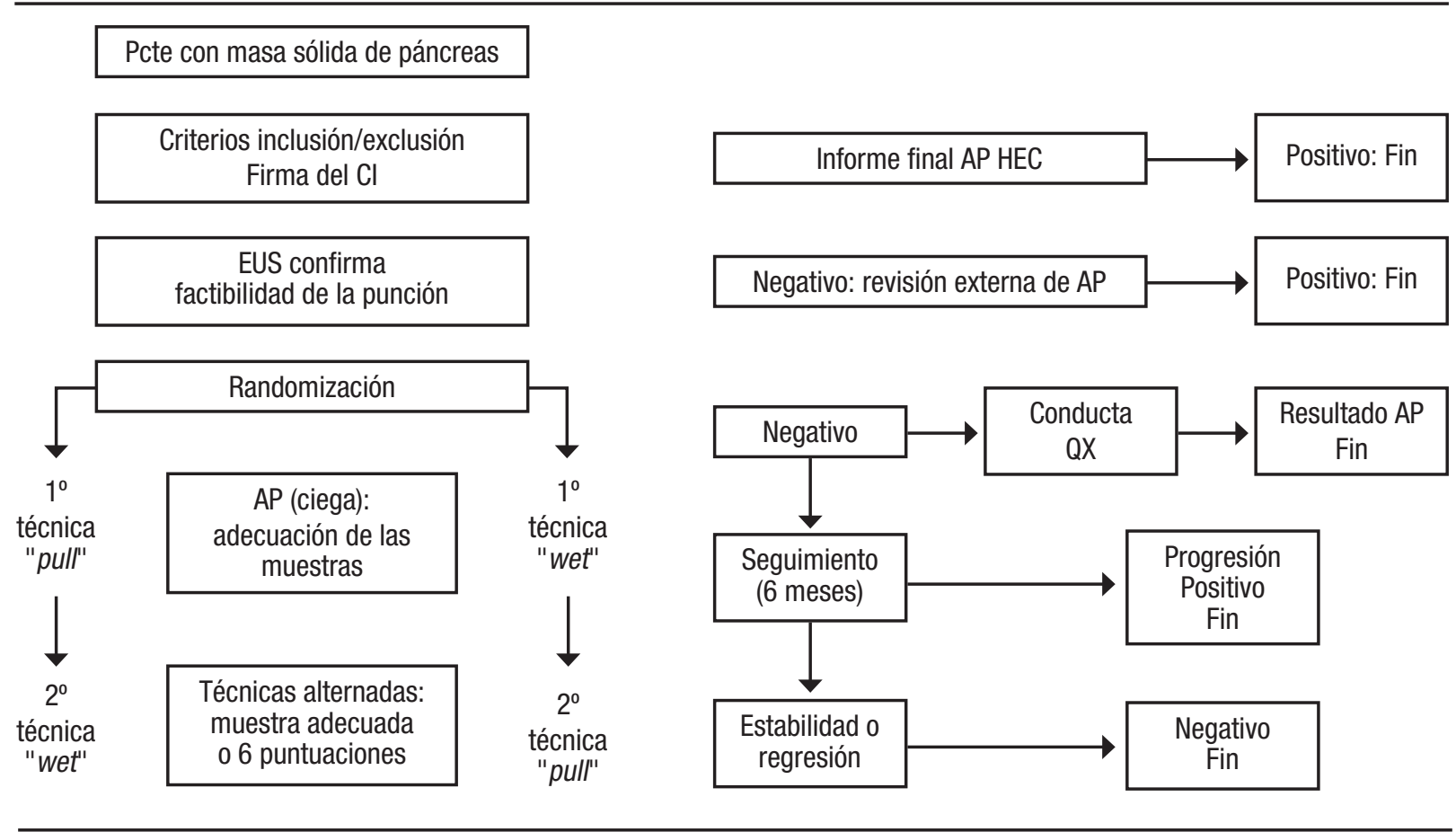

Cl: consentimiento informado; EUS: ecoendoscopia; AP: anatomía patológica; HEC: Hospital El Cruce; QX: quirúrgica. 
Criterios de inclusión y exclusión. Los criterios de inclusión fueron: pacientes mayores de 18 años con una masa sólida del páncreas.

Los criterios de exclusión fueron: coagulopatía (RIN $>1,5$ o plaquetas $<50000 / \mathrm{mm}^{3}$ ), lesiones quísticas o sólido-quísticas del páncreas, adenopatías retroperitoneales sin masa pancreática, mujeres embarazadas y los pacientes con dificultades para su seguimiento.

Cumplidos los criterios de inclusión y firmado el consentimiento, se les realizó la USE utilizando como primera técnica de punción la que correspondía según la lista de aleatorización. Se alternaron uno y otro método hasta que el citopatólogo detectó la presencia de material suficiente para el diagnóstico (definido como aquel que posee la suficiente cantidad y calidad de células que nos permite arribar a un diagnóstico de certeza) o hasta la $6^{\text {ta }}$ punción (momento a partir del cual aumentan los riesgos de complicaciones sin incrementar el rédito diagnóstico). El número mínimo de punciones fue dos: una de cada técnica.

El ecoendoscopista que realizó las punciones $(\mathrm{HJH})$ es un experto con más de 3000 USE y más de 500 PAAF realizadas.

Procedimientos. USE + PAAF: Cada paciente se posicionó en decúbito lateral izquierdo sin intubación endotraqueal. La administración de anestésicos estuvo a cargo de un especialista. No se indicó profilaxis antibiótica. Se utilizó un ecoendoscopio lineal con frecuencia de 7,5-12 MHz (EG-530UT, procesador SU-7000, Fujinon, Japón). Se exploró el páncreas, la vía biliar, la región ampular y el hígado para la identificación de lesiones y adenopatías. Se evaluó la masa pancreática describiendo el tamaño, la localización, el borde y el compromiso vascular. Se realizaron todas las punciones con una aguja de 22 G (EchoTip Ultra HD® de Cook Endoscopy o Expect ${ }^{\circledR}$ de Boston Scientific Corporation.). Se utilizó la técnica de fanning o abanico durante la punción. ${ }^{9}$

1)Técnica húmeda o wet: se retiró el estilete de la aguja antes de realizar la punción. Se inyectaron $5 \mathrm{ml}$ de solución fisiológica dentro del canal de la aguja. Una vez punzada la masa, se conectó una jeringa al vacío de $10 \mathrm{ml}$ y se abrió generando una succión permanente mientras se realizaban 20 pequeños movimientos de inserción y retirada. Una vez terminada la maniobra, se cerró la jeringa al vacío y se retiró toda la aguja.

2)Técnica por capilaridad o pull: la punción de la masa pancreática se realizó con el estilete colocado dentro de la aguja. Cuando se punzó la lesión, el estilete se retiró lentamente realizando 20 movimientos pequeños de inserción y retirada, sin salir de la masa.

Citología e histología: El material aspirado se extrajo de la aguja de punción insuflando aire con una jeringa. El examen in situ se realizó con la muestra de cada una de las pasadas. El material se recolectó en un portaobjetos para citología y el coágulo o bloque celular se fijó en formol buffer al 10\%. Se realizó la tinción de las muestras de citología con azul de toluidina para determinar la presencia de material suficiente para el análisis. El resto se fijó en alcohol $96^{\circ}$ para su posterior evaluación citológica utilizando la tinción de Papanicolaou. Las muestras de bloque celular se incluyeron en parafina. Se realizaron cortes con el micrótomo y tinción con hematoxilina-eosina para el análisis histológico e inmunohistoquímica a criterio del citopatólogo. Se utilizó la terminología y la nomenclatura estandarizada para la citología pancreático-biliar propuesta por la Sociedad de $\mathrm{Pa}-$ panicolaou: ${ }^{10,11}$ 1) muestra acelular, parénquima normal o contaminante cuyo diagnóstico es incierto; 2) pancreatitis aguda, crónica, autoinmune, tejido pancreático normal; negativo para malignidad; 3 ) anormalidades arquitecturales y citológicas no consistentes con la normalidad o los cambios reactivos; insuficiente para clasificarlas como sospechosa o neoplásica; 4) el espécimen citológico es suficientemente celular y representativo (con o sin contexto clínico, imágenes y estudios auxiliares) para ser diagnosticado como neoplásico (benigno y otras neoplasias); 5) células displásicas sospechosas de malignidad; 6) células neoplásicas malignas.

En cada muestra se evaluó la adecuación del espécimen y se consideraron las siguientes variables: ${ }^{8,12}$

- Celularidad (incluye los tipos celulares: normales y patológicos): 0 = acelular; 1 = escasa cantidad de células; 2 = moderada cantidad de células; 3 = gran cantidad de células.

- Sangre contaminante: 0 = sin sangre; 1 = contaminada por células de glóbulos rojos; 2 = presencia de coágulos de sangre.

- Adecuación del espécimen para el diagnóstico: $0=\mathrm{el}$ citopatólogo no puede realizar el diagnóstico porque la muestra no es adecuada; 1 = el citopatólogo puede realizar el diagnóstico ya que la muestra es adecuada.

Diagnóstico citopatológico final: combinación de la citología y el bloque celular.

Diagnóstico médico definitivo: como no todos los pacientes fueron sometidos a una resección quirúrgica que sería el patrón de oro del diagnóstico ideal, nosotros definimos el diagnóstico final de cada paciente basados en: a) resultados citológicos o histológicos de la punción; b) en los casos en que el paciente fue operado, se tuvo en cuenta la anatomía patológica de la pieza quirúrgica; c) en los casos en los que la PAAF no fue diagnóstica y el paciente no fue operado, la evolución clínica-imagenológica y la progresión o no de la enfermedad determinaron el diagnóstico final con un seguimiento mínimo de 6 meses posteriores a la USE-PAAF. 
Recolección de datos. La edad y el sexo, los síntomas, los hábitos tóxicos, los antecedentes patológicos, el laboratorio, los hallazgos de la USE, la cantidad de punciones realizadas y la técnica utilizada, el resultado de la citología e histología de la PAAF, la evolución y el diagnóstico definitivo. Todas las variables se recolectaron en un formulario electrónico de REDCap. ${ }^{13}$

Análisis estadístico. Las variables categóricas se expresaron en números absolutos y porcentajes; las variables continuas, en media y desvío estándar, mediana, intervalo de confianza $95 \%$ y rango, según corresponda.

Las comparaciones entre ambos métodos de punción respecto a la celularidad, la sangre contaminante y la adecuación para el diagnóstico se realizaron mediante un test no paramétrico de comparación de medias de muestras pareadas: el Wilcoxon signed rank test. Los resultados se expresaron en porcentajes.

La sensibilidad, la especificidad, el valor predictivo positivo y negativo, la precisión diagnóstica y las razones de verosimilitud positiva y negativa de cada técnica de PAAF se calcularon aplicando las definiciones estándares y utilizando una tabla de contingencias.

Para tener en cuenta el rol del azar en los acuerdos entre las técnicas y el estándar, se calculó el índice kappa ponderado para la sensibilidad, que es método estadístico expuesto por Díaz Arce et al. ${ }^{14}$ Para su interpretación se utilizó la escala de Landis y Koch citada en Fleiss et al. ${ }^{15}$

Se comparó el rendimiento diagnóstico de ambas técnicas con la prueba de $\mathrm{McNemar}$.

Las variables con $p<0,05$ de doble cola fueron consideradas estadísticamente significativas.

En el procesamiento de los datos se utilizó el programa SPSS versión 20.0.

Cálculo de N. Según estudios previos, la prevalencia del cáncer de páncreas en esta población sería del 88\% (82/93) con una sensibilidad de la USE-PAAF del 79\%. ${ }^{7}$ Para estimar una sensibilidad del $80 \%$ con un nivel de confianza de 95\%, una hemiamplitud del intervalo de confianza de $\pm 15 \%$ y considerando una prevalencia del $88 \%$, se necesitan incluir a 32 pacientes. Debido a que durante el seguimiento podría producirse una pérdida de los pacientes estimada en el $20 \%$, calculamos que se necesitarían incluir en total a 40 pacientes. La estimación del tamaño de la muestra se realizó utilizando las fórmulas de Jones et al. ${ }^{16}$

Consideraciones éticas. El trabajo se realizó en cumplimiento de la declaración de Helsinki. Fue aprobado por el Departamento de Investigación y el Comité de Ética del Hospital de Alta Complejidad El Cruce, con fecha del 11 de diciembre de 2018 y bajo el registro 030/2018.

\section{Resultados}

En el período de un año se inscribieron 41 pacientes. Veinticuatro fueron de sexo masculino $(58,5 \%)$ y la edad promedio fue 63,6 años (DS \pm 13 años) (Tabla 1).

No se presentaron complicaciones vinculadas al procedimiento.

Los síntomas que manifestaban los pacientes al momento de inscribirse fueron: pérdida de peso en 29 sujetos $(71 \%)$, ictericia y coluria en $25(61 \%)$, dolor abdominal en $21(51,2 \%)$ y diarrea crónica en $5(12,2 \%)$.

En relación con los hábitos tóxicos, 7 pacientes manifestaron mantener un consumo de alcohol (> $80 \mathrm{gr} /$ día) y 17 sujetos, consumo de tabaco (> 20 pack-year). Con respecto a los antecedentes más relevantes, 7 pacientes tenían diagnóstico de diabetes (5 de tipo II, de los cuales 3 fueron diagnosticados dentro de los 12 meses), 2 tuvieron un carcinoma renal de células claras (con tratamiento quirúrgico) y 3 presentaban antecedentes de pancreatitis aguda.

En la USE de todos los pacientes se observó una masa pancreática sólida cuyo tamaño variaba entre 10 y $70 \mathrm{~mm}$ de diámetro. La mayoría de estas lesiones se localizaba en la cabeza $(85,4 \%)$, presentaban un compromiso vascular (arterial o venoso) en el 71\%, adenomegalias en el $61 \%$, nódulos hepáticos compatibles con metástasis en el 12,2\% y ascitis en el 14,6\% de los casos (Tabla 2).

Diagnóstico final. El resultado citopatológico de las muestras de la USE-PAAF fue positivo para neoplasia en 29 pacientes y negativo en 12 . De los pacientes con resultado positivo, 27 tenían un adenocarcinoma de páncreas, 1 se diagnosticó como tumor estromal gastrointestinal (localizado en el cuerpo pancreático) y otro presentaba una metástasis de un carcinoma renal (cuyo tumor primario había sido resecado 16 años antes).

Los 12 pacientes con resultados de la PAAF negativos fueron seguidos por 6 meses o más, excepto una paciente que falleció 2 meses después de la punción por la progresión del tumor pancreático. En 5 de estos casos, se arribó a un diagnóstico de malignidad: por la progresión de la enfermedad neoplásica (con extensión a otros órganos y carcinomatosis peritoneal), por una resección quirúrgica o por una biopsia intraoperatoria positiva. El sexto paciente se diagnosticó con un linfoma pancreático a través de una biopsia ganglionar periférica (con reducción del tamaño de la masa pancreática luego del tratamiento quimioterápico indicado por oncohematología). En el séptimo caso, con un antecedente de carcinoma renal operado, se diagnosticó una metástasis del tumor renal mediante una biopsia percutánea. 
Tabla 1. Datos demográficos, antecedentes personales y resultados del laboratorio

\begin{tabular}{|c|c|c|c|c|c|}
\hline Variables & & Total & $\begin{array}{l}\text { Inicio con } \\
\text { técnica wet }\end{array}$ & $\begin{array}{l}\text { Inicio con } \\
\text { técnica pull }\end{array}$ & $p$ \\
\hline Pacientes & & 41 & 22 & 19 & NA \\
\hline \multirow[t]{2}{*}{ Sexo } & Femenino & $17(41,5 \%)$ & 10 & 7 & \multirow{2}{*}{0,28} \\
\hline & Masculino & $24(58,5 \%)$ & 12 & 12 & \\
\hline \multirow[t]{3}{*}{ Edad } & Media & 63,6 años & 65,3 & 61,6 & 0,28 \\
\hline & DS & 13 años & 13,7 & 12,3 & \multirow{2}{*}{ NA } \\
\hline & Rango (años) & $34-91$ & $34-91$ & $42-77$ & \\
\hline \multirow[t]{7}{*}{ Antecedentes patológicos } & Diabetes tipo I & $2(5 \%)$ & 1 & 1 & 1 \\
\hline & Diabetes tipo ॥ & $5(12,2 \%)$ & 3 & 2 & 1 \\
\hline & Anemia crónica & $8(19,5 \%)$ & 6 & 2 & 0,25 \\
\hline & Pancreatitis aguda & $3(7,3 \%)$ & 2 & 1 & 1 \\
\hline & Cáncer de riñón & $2(5 \%)$ & 1 & 1 & 1 \\
\hline & Cáncer de mama & $1(2,4 \%)$ & 1 & 0 & 1 \\
\hline & Cirrosis enólica & $1(2,4 \%)$ & 1 & 0 & 1 \\
\hline \multirow[t]{7}{*}{ Análisis de sangre (media \pm DS) } & TGO (U/I) & $73,3 \pm 87$ & $58 \pm 68$ & $92,8 \pm 106$ & 0,15 \\
\hline & TGP (U/l) & $123,3 \pm 182,1$ & $96 \pm 179$ & $158 \pm 187$ & 0,12 \\
\hline & FAL (U/I) & $617 \pm 542$ & $536,7 \pm 556$ & $697 \pm 533$ & 0,14 \\
\hline & $\mathrm{Bt}(\mathrm{mg} / \mathrm{dl})$ & $5 \pm 5,7$ & $5,5 \pm 5$ & $5 \pm 5,5$ & 0,6 \\
\hline & $\mathrm{Bd}(\mathrm{mg} / \mathrm{dl})$ & $4,1 \pm 4,5$ & $5 \pm 2,4$ & $5,4 \pm 4,2$ & 0,64 \\
\hline & CEA (ng/ml) & $11,3 \pm 27$ & $17,8 \pm 36,7$ & $11 \pm 19$ & 0,39 \\
\hline & CA 19-9 (ng/ml) & $699,7 \pm 1380$ & $605 \pm 1200$ & $701 \pm 1400$ & 0,3 \\
\hline
\end{tabular}

DS: desvío estándar; TGO: transaminasa glutámico oxalacética; TGP: transaminasa glutámico pirúvica; FAL: fosfatasa alcalina; Bt: bilirrubina total; Bd: bilirrubina directa; CEA: antígeno carcinoembrionario; CA 19-9: cáncer antigen 19.9; NA: no aplica.

Tabla 2. Hallazgos de la USE y características de las lesiones pancreáticas

\begin{tabular}{|c|c|c|c|c|c|}
\hline Hallazgos de la USE & & Resultados & $\begin{array}{l}\text { Inicio con } \\
\text { técnica wet }\end{array}$ & $\begin{array}{l}\text { Inicio con } \\
\text { técnica pull }\end{array}$ & $p$ \\
\hline Tamaño (mm) (media \pm DS) & & $36,6 \pm 13,2$ & $38 \pm 14,3$ & $34 \pm 12$ & 0,4 \\
\hline \multirow[t]{4}{*}{ Localización de la masa pancreática } & Cabeza & $35(85,4 \%)$ & 17 & 18 & 0,5 \\
\hline & Proceso uncinado & $8(19,5 \%)$ & 4 & 4 & 0,5 \\
\hline & Cuerpo & $10(24,4 \%)$ & 7 & 3 & 0,5 \\
\hline & Cola & $1(2,4 \%)$ & 1 & 0 & 0,5 \\
\hline \multirow[t]{4}{*}{ Compromiso vascular } & Tronco celíaco & $9(21,9 \%)$ & NA & NA & NA \\
\hline & $\begin{array}{l}\text { Arteria mesentérica } \\
\text { superior }\end{array}$ & $15(36,6 \%)$ & NA & NA & NA \\
\hline & $\begin{array}{l}\text { Vena porta y vena } \\
\text { mesentérica superior }\end{array}$ & $23(56,1 \%)$ & NA & NA & NA \\
\hline & Arteria hepática & $6(14,6 \%)$ & NA & NA & NA \\
\hline Adenomegalias & & $25(61 \%)$ & NA & NA & NA \\
\hline Metástasis hepáticas & & $5(12,2 \%)$ & NA & NA & NA \\
\hline Ascitis & & $6(14,6 \%)$ & NA & NA & NA \\
\hline
\end{tabular}

NA: no aplica. 
Finalmente, los 5 pacientes restantes, cuyas PAAF guiadas por la USE fueron negativas para neoplasias, fueron seguidos clínicamente y con estudios por imágenes durante más de 6 meses (media: 9 meses, rango: 6 a 15 meses). En 3 de estos casos, la clínica, el tamaño y las características de la masa no tuvieron cambios y se consideraron como masas inflamatorias asociadas a una pancreatitis crónica (con hallazgos de signos de inflamación crónica o calcificaciones en el resto del páncreas en la USE o RMN). En un paciente se resecó la masa y en otro se tomó una biopsia quirúrgica diagnosticándose, en ambos, un proceso inflamatorio crónico. Ninguno tuvo serología IgG 4 positiva.

El diagnóstico final contra el que se compararon ambas técnicas de la PAAF fue: 36 pacientes con neoplasia de páncreas (32 con adenocarcinoma) y 5 pacientes con masa inflamatoria (Tabla 3).

Tabla 3. Diagnóstico final

\begin{tabular}{lc}
\hline Diagnóstico & N \\
\hline Adenocarcinoma de páncreas & 32 \\
GIST & 1 \\
Linfoma & 1 \\
Metástasis de carcinoma renal & 2 \\
Masa inflamatoria & 5
\end{tabular}

Rendimiento diagnóstico de la USE-PAAF: método wet vs pull. De los 41 pacientes inscriptos, 22 fueron aleatorizados a realizarse la primera punción con la técnica húmeda o wet y 19 con la técnica capilaridad o pull. Se realizaron, en total, 140 punciones con un promedio de 3,4 punciones por paciente (rango: 2 y 6 punciones). El promedio de punciones en el grupo de pacientes que inició con la técnica wet fue de 3,73 (DS \pm 0,83) (IC 95\%; $3,36-4,09)$ y en el grupo de pacientes que inició con técnica pull fue de 3,05 (DS $\pm 0,85$ ) (IC 95\%; 2,64-3,46), $\sin$ diferencias significativas. Del total de punciones, 73 fueron con el método húmedo y 67 con la técnica de capilaridad.

La sensibilidad, la especificidad, el valor predictivo positivo y negativo, la precisión diagnóstica de la PAAF guiada por la USE (sumando los resultados de la técnica pull y wet) fueron: $80,6 \% ; 100 \% ; 100 \% ; 41,7 \%$ y $82,9 \%$, respectivamente (Tabla 4 ).

La sensibilidad, la especificidad y la precisión diagnóstica esperadas fueron: 70,7\%; 29\% y 65,7\%. Los valores del estadístico kappa ponderado respectivos fueron: 0,34 (pobre); 1 (muy bueno) y 0,50 (moderado).
Analizando las punciones que utilizaron la técnica wet, la sensibilidad, la especificidad, el valor predictivo positivo y negativo y la precisión diagnóstica fueron: $58,3 \%$; $100 \% ; 100 \% ; 25 \%$ y $63,4 \%$, respectivamente. La razón de verosimilitud LR+ fue mayor a 10 , debido a la especificidad de $100 \%$, y LR- fue 0,42 (Tabla 5).

La sensibilidad, la especificidad y la precisión diagnóstica esperadas fueron: $51,2 \%, 49 \%$ y $50,9 \%$ y los valores del estadístico kappa ponderado respectivos fueron: 0,15 (pobre); 1 (muy bueno) y 0,25 (pobre).

El resultado de las punciones con el método de pull fue: $75 \%, 100 \%, 100 \%, 35,7 \%$ y $78,1 \%$, respectivamente. La razón de verosimilitud $\mathrm{LR}+$ fue mayor a 10 por la especificidad de $100 \%$ y LR- fue 0,25 (Tabla 4).

La sensibilidad, la especificidad y la precisión diagnóstica esperadas fueron: $65,9 \%$; 34,0\% y $62,0 \%$. Los valores del estadístico kappa ponderado respectivos fueron: 0,27 (pobre); 1 (muy bueno) y 0,42 (moderado).

Comparando el rendimiento diagnóstico entre ambas técnicas de punción, no hubo una diferencia estadísticamente significativa (test de McNemar, $p=0,388$ ). La concordancia entre ambas técnicas en resultados positivos fue del 40,3\%; en resultados negativos fue del 40,3\%; mientras que la discordancia diagnóstica fue del 19,4\%.

De las 140 punciones, se obtuvo un coágulo o bloque celular para tinción con $\mathrm{H} \& \mathrm{E}$ en 123 (87,8\%). No hubo una diferencia significativa entre las dos técnicas de punción para obtener el bloque celular (húmedo 87,7\% y capilaridad $88 \%, p=0,85$ ).

Calidad de la muestra. Respecto a la celularidad del espécimen, teniendo a mayor puntaje, mayor cantidad de células para el diagnóstico ( 0 a 3 ), tanto en muestras de citología como en el bloque celular, no se observaron diferencias significativas entre las técnicas de punción ( $p=0,84$ y 0,61, respectivamente) (Tabla 5).

La sangre contaminante de la muestra puede afectar el rendimiento diagnóstico, teniendo a mayor puntaje, mayor efecto negativo ( 0 a 2$)$. No hubo diferencias, entre las técnicas, en las muestras de citología $(p=0,89)$ y tampoco en las del bloque celular $(p=0,08)$ (Tabla 6).

La adecuación de las muestras de citología para el diagnóstico fue similar en ambas técnicas de punción (wet $=57,5 \%$ y pull $=56,7 \% ; p=0,94)$. Tampoco hubo diferencias en las muestras del bloque celular (wet $=75 \%$ y pull $=66,1 \% ; p=0,38)$.

Analizando la sensibilidad acumulada de cada punción, independientemente de cuál fue la técnica utilizada, observamos que desde la primera hasta la cuarta punción se incrementó la sensibilidad (55,5\%; 66,7\%; $77,8 \%$ y $80,5 \%$, respectivamente). Esto no sucedió con la quinta y sexta. 
Tabla 4. Resultados del rendimiento diagnóstico de las punciones por la USE y comparación entre las técnicas por capilaridad y humeda

\begin{tabular}{|c|c|c|c|c|c|c|}
\hline Método de punción & $\begin{array}{c}\mathrm{S} \\
\text { media (IC 95\%) }\end{array}$ & $\begin{array}{c}\mathrm{E} \\
\text { media (IC 95\%) }\end{array}$ & $\begin{array}{c}\text { VPP } \\
\text { media (IC 95\%) }\end{array}$ & $\begin{array}{c}\text { VPN } \\
\text { media (IC 95\%) }\end{array}$ & $\begin{array}{c}\mathrm{P} \\
\text { media (IC 95\%) }\end{array}$ & $p$ \\
\hline $\begin{array}{l}\text { USE + PAAF }(\%) \\
\text { (capilaridad + húmeda) }\end{array}$ & $80,6(63,4-91,2)$ & $100(46,3-98,1)$ & $100(85,4-99,69)$ & $41,7(16,5-71,4)$ & $82,9(67,3-92,3)$ & \\
\hline Técnica húmeda (\%) & $58,3(40,9-74,1)$ & $100(46,3-98,1)$ & $100(80,7-99,6)$ & $25(9,6-49,4)$ & $63,4(46,9-77,4)$ & 0,388 \\
\hline Técnica por capilaridad (\%) & $75(57,5-87,3)$ & $100(46,3-98,1)$ & $100(84,5-99,7)$ & $35,7(13,9-64,4)$ & $78,1(61,9-88,9)$ & 0,388 \\
\hline
\end{tabular}

S: sensibilidad; E: especificidad; VPP: valor predictivo positivo; VPN: valor predictivo negativo; P: precisión diagnóstica; IC: intervalo de confianza.

Tabla 5. Celularidad del espécimen en las muestras de citología y del bloque celular

\begin{tabular}{|c|c|c|c|c|c|c|}
\hline Muestras de citología & & 0 & 1 & 2 & 3 & $p$ \\
\hline \multirow[t]{2}{*}{ Técnica de punción } & Pull (\%) & 15,1 & 63 & 17,8 & 4,1 & 0,84 \\
\hline & Wet $(\%)$ & 25,4 & 49,3 & 22,4 & 3 & 0,84 \\
\hline Bloque celular & & 0 & 1 & 2 & 3 & $p$ \\
\hline \multirow[t]{2}{*}{ Técnica de punción } & Pull (\%) & 12,5 & 54,7 & 25 & 7,8 & 0,61 \\
\hline & Wet (\%) & 18,6 & 54,2 & 23,7 & 3,4 & 0,61 \\
\hline
\end{tabular}

0: acelular; 1: escasa cantidad de células; 2: moderada cantidad de células; 3: gran cantidad de células.

Tabla 6. Contaminación con sangre. Muestra de citología y del bloque celular

\begin{tabular}{|c|c|c|c|c|c|}
\hline Muestras de citología & & 0 & 1 & 2 & $p$ \\
\hline \multirow[t]{2}{*}{ Técnica de punción } & Pull (\%) & 17,8 & 63 & 19,2 & 0,89 \\
\hline & Wet $(\%)$ & 20,9 & 61,2 & 17,9 & 0,89 \\
\hline Bloque celular & & 0 & 1 & 2 & $p$ \\
\hline \multirow[t]{2}{*}{ Técnica de punción } & Pull (\%) & 37,5 & 56,3 & 6,3 & 0,08 \\
\hline & Wet (\%) & 37,3 & 50,8 & 11,9 & 0,08 \\
\hline
\end{tabular}

0: sin sangre; 1: contaminada por células de glóbulos rojos; 2: presencia de coágulos de sangre.

\section{Discusión}

El cáncer de páncreas es una causa significativa de morbilidad y mortalidad. En la Argentina, se estiman 4878 casos diagnosticados en $2018 .{ }^{17}$ A pesar de su baja prevalencia (5-8 casos/10 habitantes), ${ }^{5}$ representa uno de los tumores malignos con menor sobrevida $(<5 \%$ a los 5 años). ${ }^{18}$ Por lo tanto, el diagnóstico temprano y preciso es vital para mejorar la eficacia de la intervención terapéutica.

Casi todas las lesiones sólidas de este órgano son adenocarcinomas. Sin embargo, hay otras etiologías cuyas imágenes en las TC y RMN no son suficientemente claras para el diagnóstico certero. Por lo tanto, el diagnóstico anatomopatológico adquiere relevancia para optimizar el manejo de estos pacientes.

La USE se ha posicionado como una herramienta imprescindible en el proceso de diagnóstico, estadificación y punción de las lesiones sólidas de páncreas, cumpliendo con todas estas funciones en un mismo procedimiento. La USE-PAAF permite el diagnóstico citopatológico de las lesiones con una elevada sensibilidad (85-91\%) y especificidad (94-98\%), una buena tolerancia, una baja tasa de complicaciones $(<2 \%)$ y un ínfimo riesgo de diseminación a lo largo del tracto de la punción. 3, 4, 19, 20, 21

Se han descripto diferentes técnicas de punción con el objetivo de encontrar el método que permita obtener el 
mayor rendimiento diagnóstico. Estas variantes son: utilizar o no la succión, el uso o no del estilete, la técnica del abanico y el diámetro de la aguja. ${ }^{22}$ Incluso se han diseñado agujas que podrían obtener una biopsia de la lesión. ${ }^{23,24}$ Sin embargo, otros factores muy importantes que influyen en el rendimiento son: la experiencia del ecoendoscopista y del citopatólogo, la manipulación adecuada del espécimen y la presencia del citopatólogo en la sala. ${ }^{25}$

En los últimos años se han evaluado dos nuevas técnicas de punción: pull y wet. El rendimiento diagnóstico de estas nuevas técnicas fue comparado con el método de la punción estándar. En el estudio de Nakai et al., utilizando aguja de $25 \mathrm{G}$, obtuvieron una sensibilidad diagnóstica del $90 \%$ con el método de aspiración por capilaridad o pull comparado con el 67,9\% del método estándar. Sin embargo, no observaron diferencias al utilizar la aguja de 22 G (79,2\% vs. $76 \%$, respectivamente). ${ }^{7}$ Respecto a la técnica wet, Attam et al. obtuvieron un incremento significativo de la sensibilidad respecto al método estándar con la aguja de $25 \mathrm{G}(85,5 \%$ vs. $75,2 \%) .{ }^{8}$ A pesar de que estas nuevas técnicas han mostrado un mayor rendimiento respecto al método estándar, no hay estudios publicados que las comparen entre sí. Fue este el objetivo de nuestro estudio.

En nuestros resultados, obtuvimos un rendimiento adecuado de las PAAF guiadas por la USE (como técnica diagnóstica en su conjunto). El desempeño resultó muy bueno para la especificidad y relativamente bueno para la sensibilidad, lo que concuerda con los estudios publicados. ${ }^{20}$

No hallamos diferencias en cuanto a la calidad de la muestra entre ambas técnicas de punción. Sin embargo, observamos que la sensibilidad del método de pull fue mayor que la wet, aunque sin diferencia estadísticamente significativa, y que combinadas incrementan aún más la sensibilidad. Con la técnica wet, hubo $41,7 \%$ de falsos negativos, con el método de pull $25 \%$ y combinadas 19,4\%. Por lo tanto, si bien las diferencias en performance diagnóstica no fueron estadísticamente significativas, probablemente debido al número de los pacientes inscriptos, se podría tener una significancia clínica a la hora de optar por la metodología de la PAAF a utilizar en la práctica diaria. Así mismo, otra alternativa sería combinar ambos métodos, como lo hicimos en nuestro protocolo.

Una de las limitaciones de este estudio es el número de los pacientes involucrados, el cual resultó factible a expensas de amplios intervalos de confianza. Si bien existen publicaciones que reportan sensibilidades cercanas al $65 \%$, dado que la anatomía patológica define la conducta, serían deseables valores de sensibilidad mayores. ${ }^{26-29}$ Esto implicaría un número mayor de pacientes. Otra limitante es la falta de un gold standard contundente como la pieza quirúrgica o la histología de las muestras obtenidas mediante una cirugía. Debido a que en la práctica habitual no todos los pacientes son candidatos a la resección quirúrgica y se debe basar el diagnóstico final de muchos en la evolución clínica e imagenológica, creemos que nuestros resultados se acercan a la realidad y son comparables con la práctica diaria, por lo que en este aspecto se puede interpretar como una fortaleza.

La última limitante tiene que ver con la falta de registro sobre el tipo de aguja utilizada. Sin embargo, las dos principales marcas que hay en el mercado y que usamos nosotros son de muy buena calidad por lo que creemos que no es una fuente de sesgo.

Otras de las fortalezas del estudio son: la presencia del citopatólogo en la sala, el mantenimiento del ciego hasta la instancia misma del análisis de datos, la aleatorización de la técnica inicial de la punción y la decisión de punzar cada masa con ambas técnicas para disminuir la variabilidad de los resultados.

En conclusión, en este estudio no observamos diferencias en el rendimiento diagnóstico ni en la calidad de las muestras entre las técnicas de punción wet y pull. Dado que ambas técnicas son efectivas, se sugiere su utilización simultánea y alternada en las punciones de lesiones sólidas de páncreas guiadas por una USE.

Agradecimientos. Esta investigación contó con el apoyo financiero de la Sociedad Argentina de Gastroenterología a través de su beca para la investigación en gastroenterología del 2017.

\section{Aviso de derechos de autor}

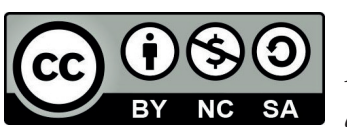

(C) 2021 Acta Gastroenterológica Latinoamericana. Este es un artículo de acceso abierto publicado bajo los términos de la Licencia Creative Commons Attribution (CC BY-NC-SA 4.0), la cual permite el uso, la distribución $y$ la reproducción de forma no comercial, siempre que se cite al autor y la fuente original.

Cite este artículo como: Curvale C, Málaga I, Rojas Saunero $P$ y col. Pull vs. wet: rendimiento diagnóstico y calidad de las muestras de las PAAF guiadas por USE en las masas sólidas del páncreas. Acta Gastroenterol Latinoam. 2021;51(1):42-51. https://doi.org/10.52787/knpg3537

\section{Referencias}

1. Tamm E, Charnsangavej C. Pancreatic cancer: current concepts in imaging for diagnosis and staging. Cancer J. 2001;7(4):298-311. 
2. Iglesias-Garcia J, Dominguez-Munoz E, Lozano-Leon A, Abdulkader I, Larino- Noia J, Antunez J, Forteza J. Impact of endoscopic ultrasound-guided fine needle biopsy for diagnosis of pancreatic masses. World J Gastroenterol. 2007;13(2):289-93.

3. Weston BR, Bhutani MS. Optimizing diagnostic yield for EUSguided sampling of solid pancreatic lesions: A technical review. Gastroenterology and Hepatology. 2013;9(6):352-63.

4. Hewitt MJ, McPhail MJW, Possamai L, Dhar A, Vlavianos P, Monahan KJ. EUS- guided FNA for diagnosis of solid pancreatic neoplasms: a meta-analysis. Gastrointest Endosc. 2012;75(2):319-31.

5. Wiersema MJ, Hawes RH, Tao LC, Wiersema LM, Kopecky KK, Rex DK, et al. Endoscopic ultrasonography as an adjunct to fine needle aspiration cytology of the upper and lower gastrointestinal tract. Gastrointest Endosc. 1992;38(1):35-9.

6. Villa NA, Berzosa M, Wallace MB, Raijman I. Endoscopic ultrasound-guided fine needle aspiration: The wet suction technique. Endoscopic Ultrasound. 2016;5:17-20.

7. Nakai Y, Isayama H, Chang KJ, Yamamoto N, Hamada T, Uchino $\mathrm{R}$, et al. Slow pull versus suction in endoscopic ultrasoundguided fine-needle aspiration of pancreatic solid masses. Dig Dis Sci. 2014;59(7):1578-85.

8. Attam R, Arain MA, Bloechl SJ, Trikudanathan G, Munigala S, Bakman Y, et al. "wet suction technique (WEST)": A novel way to enhance the quality of EUS- FNA aspirate. Results of a prospective, single-blind, randomized, controlled trial using a 22-gauge needle for EUS-FNA of solid lesions. Gastrointest Endosc. 2015;81(6):1401-7.

9. Bang JY, Magee SH, Ramesh J, Trevino JM, Varadarajulu S. Randomized trial comparing fanning with standard technique for endoscopic ultrasound-guided fine-needle aspiration of solid pancreatic mass lesions. Endoscopy. 2013;45(6):445-50.

10. Pitman MB, Layfield LJ. Guidelines for pancreaticobiliary cytology from the Papanicolaou Society of Cytopathology: A review. Cancer Cytopathology. 2014;122(6):399-411.

11. Pitman MB, Centeno BA, Ali SZ, Genevay M, Stelow E, Mino-Kenudson M, et al. Standardized terminology and nomenclature for pancreatobiliary cytology: The Papanicolaou Society of Cytopathology Guidelines. Diagn Cytopathol. 2014;42(4):338-50.

12. Wee E, Lakhtakia S, Gupta R, Sekaran A, Kalapala R, Monga $A$, et al. Endoscopic ultrasound guided fine-needle aspiration of lymph nodes and solid masses: Factors influencing the cellularity and adequacy of the aspirate. J Clin Gastroenterol. 2012;46(6):487-93.

13. Harris PA, Taylor R, Thielke R, Payne J, Gonzalez N, Conde JG. Research electronic data capture (REDCap)-A metadata-driven methodology and workflow process for providing translational research informatics support. J Biomed Inform. 2009;42(2):377-81.

14. Díaz Arce D, Beltrán Carreño JP, Cueva Sarmiento JE. ¿¿Son suficientes los indicadores del rendimiento de una prueba o test diagnóstico para evaluar su desempeño?. Rev Cuba Med Gen Integr. 2018;34(3):94-109.

15. Fleiss JL, Levin B, Paik MC. The Measurement of Interrater Agreement. En: Stat Methods Rates Proportions. 2004;598-626.
16. Jones SR, Carley S, Harrison M. An introduction to power and sample size estimation. Emerg Med J. 2003;20(5):453-8.

17. Instituto Nacional del Cáncer, (INC). Boletín de vigilancia epidemiológica. Minist Salud la Nac. 2018;1-50.

18. Jemal A, Bray F, Center MM, Ferlay J, Ward E, Forman D. Global cancer statistics. CA Cancer J Clin. 2011;61(2):69-90.

19. ASGE Standards of Practice Committee; Early DS, Acosta RD, Chandrasekhara V, Chathadi KV, Decker GA, Evans JA, et al. Adverse events associated with EUS and EUS with FNA. Gastrointest Endosc. 2013;77(6):839-43.

20. Banafea O, Mghanga FP, Zhao J, Zhao R, Zhu L. Endoscopic ultrasonography with fine-needle aspiration for histological diagnosis of solid pancreatic masses: A meta-analysis of diagnostic accuracy studies. BMC Gastroenterol. 2016;16(1):108.

21. Dumonceau JM, Deprez PH, Jenssen C, Iglesias-Garcia J, Larghi A, Vanbiervliet G, et al. Indications, results, and clinical impact of endoscopic ultrasound (EUS)-guided sampling in gastroenterology: European Society of Gastrointestinal Endoscopy (ESGE) Clinical Guideline - Updated January 2017. Endoscopy. 2017;49(7):695-714.

22. Wani S. Basic techniques in endoscopic ultrasound-guided fineneedle aspiration: Role of a stylet and suction. Endoscopic Ultrasound. 2014;3:17-21.

23. Bang JY, Kirtane S, Krall K, Navaneethan U, Hasan M, Hawes R, Varadarajulu S. In memoriam: Fine-needle aspiration, birth: Fine-needle biopsy: The changing trend in endoscopic ultrasoundguided tissue acquisition. Dig Endosc. 2019;31(2):197-202.

24. Wang F, Wang HL, Zhao Q. Endoscopic Ultrasound-Fine-Needle Biopsy Is Superior to Endoscopic Ultrasound-Fine-Needle Aspiration in Sampling Pancreatic Masses. Clin Gastroenterol Hepatol. 2018;16(5):785-7.

25. Matynia AP, Schmidt RL, Barraza G, Layfield LJ, Siddiqui AA, Adler DG. Impact of rapid on-site evaluation on the adequacy of endoscopic-ultrasound guided fine-needle aspiration of solid pancreatic lesions: A systematic review and meta- analysis. J Gastroenterol Hepatol. 2014;29(4):697-705.

26. Touchefeu Y, Le Rhun M, Coron E, Alamdari A, Heymann MF, Mosnier JF, et al. Endoscopic ultrasound-guided fine-needle aspiration for the diagnosis of solid pancreatic masses: The impact on patient-management strategy. Aliment Pharmacol Ther. 2009;30(10):1070-7.

27. Ryozawa S, Kitoh H, Gondo T, Urayama N, Yamashita H, Ozawa $\mathrm{H}$, et al. Usefulness of endoscopic ultrasound-guided fineneedle aspiration biopsy for the diagnosis of pancreatic cancer. J Gastroenterol. 2005;40(9):907-11.

28. Voss M, Hammel P, Molas G, Palazzo L, Dancour A, O’Toole D, Terris B, Degott C, Bernades P, Ruszniewski P. Value of endoscopic ultrasound guided fine needle aspiration biopsy in the diagnosis of solid pancreatic masses. Gut. 2000;46(2):244-9.

29. Afify AM, al-Khafaji BM, Kim B, Scheiman JM. Endoscopic Ultrasound-Guided Fine Needle Aspiration of the Pancreas. Diagnostic Utility and Accuracy. Acta Cytol. 2003; 47(3):341-8. 